\title{
Thin film description by wavelet coefficients statistics
}

\author{
JiŘí BOLDYŠ ${ }^{* \#}$ AND RUdOLF HRACH * \\ * Department of Electronics and Vacuum Physics, Faculty of Mathematics and Physics, \\ Charles University, V Holešovičkách 2, 18000 Prague 8, Czech Republic \\ $\sharp$ Institute of Information Theory and Automation, Academy of Sciences of the Czech \\ Republic, Pod vodárenskou věží 4, 18208 Prague 8, Czech Republic
}

Received XXX

\begin{abstract}
Descriptive and robust features based on wavelet transform coefficients are proposed for a multiscale thin film image analysis. The features are based on one- and two-dimensional histograms of the wavelet transform coefficients and they can be calculated for every scale of the wavelet decomposition. A one-dimensional histogram model is extended to describe also two-dimensional histograms, by means of calculating marginal histograms and by sampling the two-dimensional histograms in orientation. A computer experiment has been performed to demonstrate correspondence of the derived features to various physical phenomena.
\end{abstract}

PACS: $68.55 . J k, 02.30 . \mathrm{Qy}$

Key words: thin films, wavelet transform, descriptors, histogram model

\section{Introduction}

Thin film technology has definitely invaded our everyday lives. Variety of applications of their unique characteristics has been found in metal industry, optics, cosmetics or telecommunications.

Examination of the experimentally acquired or the growing thin films is an important task in thin film physics. Thin film imaging by a variety of modalities available nowadays is probably the most important source of information. Thin film images provide information about thin film morphology, including structure of the thin film surface.

In thin film physics, there has been attempts to describe thin films by means of image processing. There exist powerful methods, including methods of mathematical morphology, applied mainly to binary images [1, 2]. However, so far, they have been used mainly in the context of discontinuous thin film morphology and for description of simpler or idealized structures $[3,4,5]$.

In this paper, one- or two-dimensional (1D or 2D) histograms of wavelet coefficients from different wavelet transform sub-images are used to calculate features, which have simple clear meanings and which describe well the thin film morphology. Since, to our knowledge, the surface structure (texture) characteristics have not been analyzed in the thin film physics literature, the paper focuses on them. A computer experiment has been performed to demonstrate correspondence of the derived features to various physical phenomena.

$\left.{ }^{1}\right)$ Corresponding author; e-mail: boldys@utia.cas.cz 
The paper is organized as follows. Firstly, image processing background is either introduced or links to the relevant literature are provided. Secondly, the novel features and their interpretation are proposed. Afterwards, a few experiments demonstrating descriptiveness of the features are conducted and the results are discussed.

\section{Multiscale image statistics}

One of the most frequent tools for texture description are image intensity (graylevel) histograms. They contain information about appearance of certain gray levels, partially corresponding to variability in thin film images. Spatial relations between various gray levels can be captured by $2 \mathrm{D}$ histograms, called also co-occurrence matrices (CM, see [6]).

Traditionally, co-occurrence matrix is defined on a window, see e.g. [6]. In this paper, advantages of the vectorized definition are used. Given a vector of pixels $\mathbf{x}$ of an image and their gray-level values $g(\mathbf{x})$. Given a spatial relation by means of a shift $[a b]$ (in pixels), determining neighbors $\mathbf{y}$ of the pixels $\mathbf{x}$. Their gray-level values are $g(\mathbf{y})$. Pairs of gray-levels $\left\{g(\mathbf{x})_{i} g(\mathbf{y})_{i}\right\}$ for all $i$ are then coordinates to the co-occurrence matrix.

The histograms can be also calculated on other values than image intensities. Coefficients of discrete wavelet transform (DWT) are used in this paper. A thorough introduction to wavelet transform can be found, for example, in [7]. In this paper, the biorthogonal wavelet bior1.3 [8] is used, see Fig. 1. It has only one vanishing moment. Therefore, it is sensitive to an arbitrary change of image intensity (except of adding a constant). Wavelets with more vanishing moments do not respond to linear intensity changes. Thus, interpretation of their responses is more complicated. Generally, a reasonable smoothness of the wavelet is desirable, although it is not crucial for the data presented here. Since the bior1.3 wavelet is symmetrical, its response can be easily attributed to a certain point. Exact symmetry is, except of the Haar wavelet, in contradiction with orthogonality, which would have been also useful in feature extraction.

2D DWT of a thin film image can by calculated as a decomposition pyramid, transforming both coordinates independently. Thus, the coefficients can be attributed with horizontal, vertical, and diagonal direction ( $\mathrm{H}, \mathrm{V}, \mathrm{D}$, respectively) and with image approximation at particular scales of analysis, see Fig. 1.

The histograms can be then calculated on one of the sub-images or on two of them, different by orientation, scale or mutual shift, see e.g. [9]. The histogram shapes are usually very constrained, especially for images of natural scenes. The 1D histograms are often modeled by the family of generalized exponentials [10]:

$$
h(x)=K e^{-(|x| / \alpha)^{\beta}} .
$$

$x$ is the wavelet coefficient value, $\alpha$ and $\beta$ are parameters and $K$ is a normalization factor. $\alpha$ and $\beta$ describe the histogram shape and, therefore, can be used as features. The model (1) for different parameters $\alpha$ and $\beta$ is plotted in Fig. 2.

The parameter $\alpha$ determines the width of the function (1) and thus corresponds 

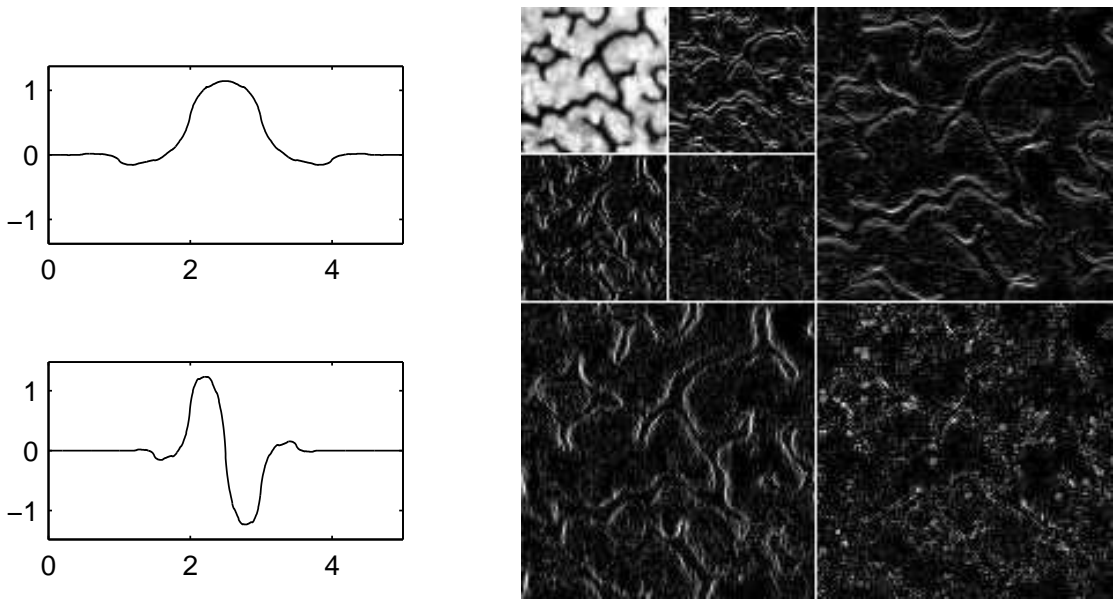

Fig. 1. Left: Scaling function (top) and wavelet (bottom) used in this paper (bior1.3). Right: 2D DWT two-level pyramid of a thin film image similar to the top left thin film image from Fig. 3. Horizontal (top right corners), vertical (bottom left corners) and diagonal (bottom right corners) detail sub-images and the two-level approximation sub-image (top left corner) are shown.
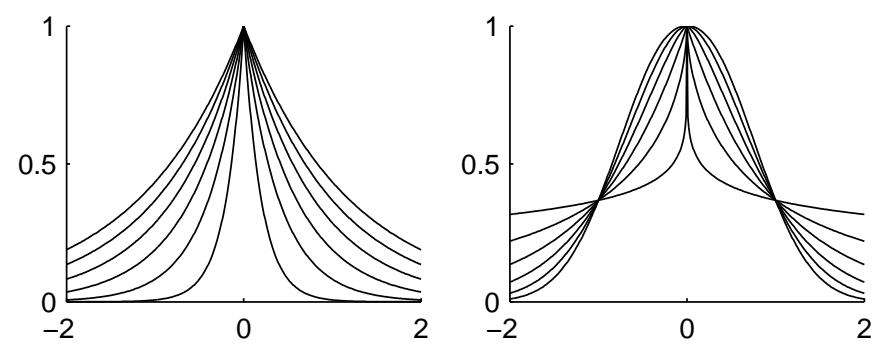

Fig. 2. Different shapes of the histogram model for evenly sampled parameters $\alpha$ (left) and $\beta$ (right).

to contrast of the measured phenomenon. For smaller $\beta$, the function exhibits a sharper peak and heavier tails. There is majority of approximately zero wavelet coefficients and enough wavelet coefficients with relatively high magnitudes in the corresponding decomposition sub-image. That roughly corresponds to flatter thin films with sharper borders, at a corresponding scale and orientation. When $\beta$ is higher, relative amount of coefficients with medium magnitudes is higher and the coefficients with large magnitudes are absent. In this case, the thin film tops are 
not flat any more. This can be, for example, due to a bias of otherwise flat structure or simply due to its round shape.

\section{Features for multiscale thin film image description}

In this paper, the generalized exponentials (1) are used to fit the $1 \mathrm{D}$ histograms and the parameters $\alpha$ and $\beta$ are used as features. The parameters $K, \alpha$ and $\beta$ from (1) are first estimated based on first and second order moments, using equations given in [11]. In some cases, this estimation exhibits certain un-robust behavior. Therefore, the simplex minimization method is used to refine the parameters. A weighted combination of absolute and relative deviations is used in the minimization criterion. The deviations are taken into account only for histogram bins with nonzero number of counts.

In case of 2D histograms, all their marginal distributions can be modeled first, and the 2D histogram model can be reconstructed afterwards, as in [12]. However, the parameters describing to model are not easy to interpret.

It has been empirically verified that behavior of the CMs in the four main directions describes them sufficiently well. Therefore, in this paper, the CMs are described by 1D histograms, which are either marginal histograms, or certain samples of the CMs in orientation. Only two more marginal histograms are thus needed, since the $1 \mathrm{D}$ histograms are the remaining two. If $x_{1}$ and $x_{2}$ are random variables, the marginal histograms corresponding to the random variables $\left(x_{1}+x_{2}\right) / \sqrt{2}$, resp. $\left(x_{1}-x_{2}\right) / \sqrt{2}$ are denoted $M 1$, resp. $M 2$.

Marginal distributions are projections of the 2D histograms. Four other 1D histograms can be obtained by making sections through the center of a CM and detecting the corresponding profiles. The $x_{1}-x_{2}$ plane is smoothly divided (sampled) in orientation by a weighting function. It averages the values in a certain distance from the center and belonging to the corresponding section in orientation. The histograms are denoted $S 0-S 3$, where $S j$ corresponds to counterclockwise rotation $j \pi / 4$ of the weighting function. More details can be found in [13].

In the actual implementation, there is no need to construct the CM to calculate the histograms. Their description by means of the parameters $\alpha$ and $\beta$ has the advantage of straightforward interpretation in the context of thin film image analysis. Sometimes, due to nature of the underlying problem, there is no justification for using the symmetrical histogram model (1). In this case, the left and the right halves of the histograms are fitted separately.

\section{Experimental results}

Four representative thin film images are transformed and the sums of the 1D histograms of the horizontal and the vertical sub-images at level four are modeled symmetrically and drawn in Fig. 3. Size of all the images used in this section is $512 \times 512$ pixels. Usage of the model (1) is justified, as well as in the later examples, by a good fit to the histograms. The histogram shapes correspond to morphology of 
the thin films. Generally, flatter and sharper images exhibit more spiky histograms.
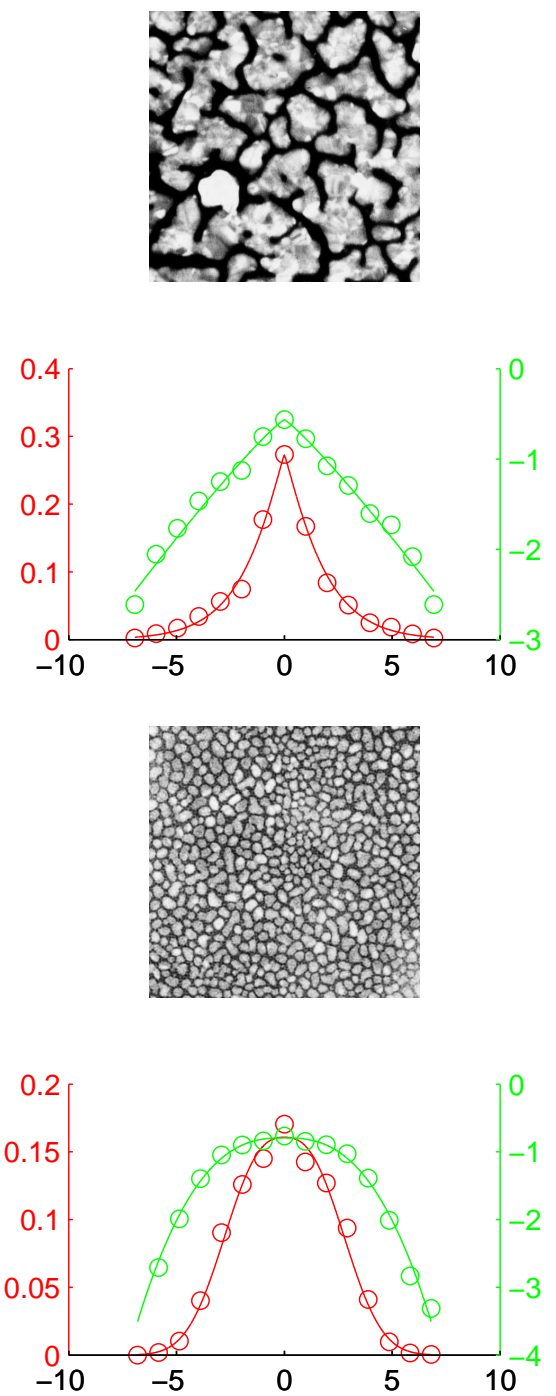
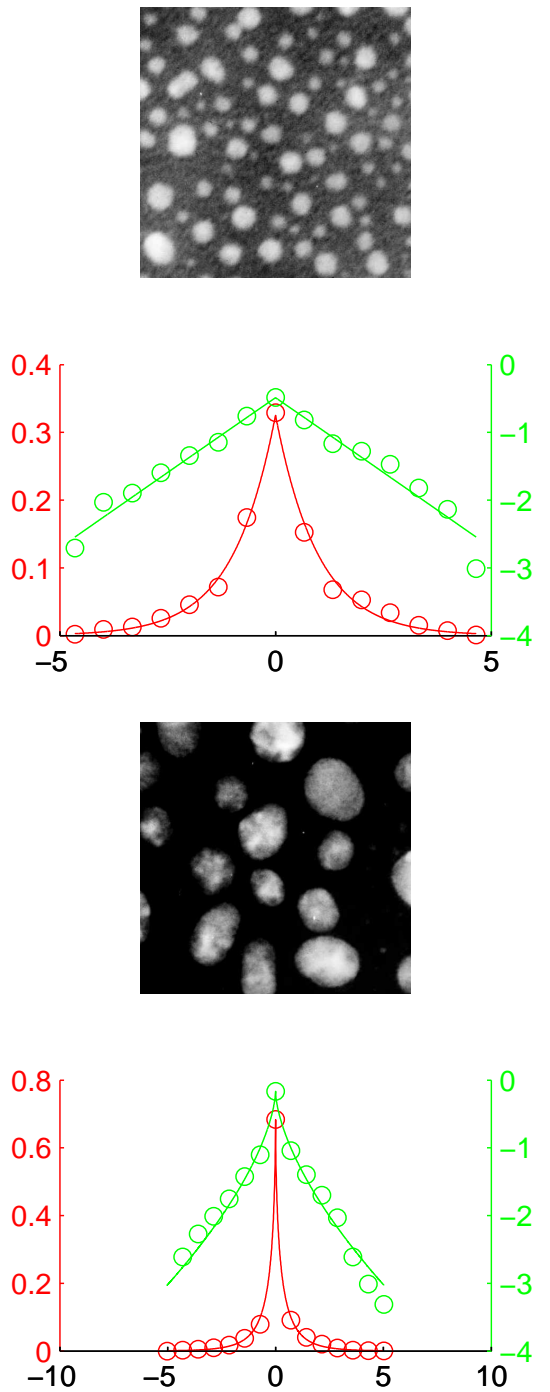

Fig. 3. One-dimensional histograms of both horizontal and vertical wavelet coefficients, modeled as symmetrical, for four representative thin films. Level four. The top curves depict the histograms in the logarithmic scale (right axes).

In the following, a physically motivated computer experiment is performed. Histograms describing the CM structure and, therefore, also thin film morphology at various scales, are used here. A thin film image containing a crystalized structure, similar to the top left thin film image from Fig. 3, was chosen. Afterwards, it was 
blurred to make the surface smoother and thus containing less sharp variations typical for crystallization. The amount of crystallization approximately modeled in this way was then assessed by means of the features proposed in Section 3. Only coefficients corresponding to the thin film top surface are used to calculate the CMs to focus the responses only to the crystallization phenomenon, see [13].

Different degrees of crystallization are modeled by convolving with a disk kernel of the diameter $2 b+1$ pixels. Thus, $b=0$ means no smoothing. Influence of the parameter $b$ on the thin film profile shape is demonstrated in Fig. 4, where the image profiles with parameters $b=0,5,10,15,20$ are drawn. It should be emphasized here, that the model of crystallization is only a simple and rough visual approximation. It does not originate from thin film physics. Our main objective is to show the correspondence between the changing structure of the thin film images with the features derived from wavelet coefficients.

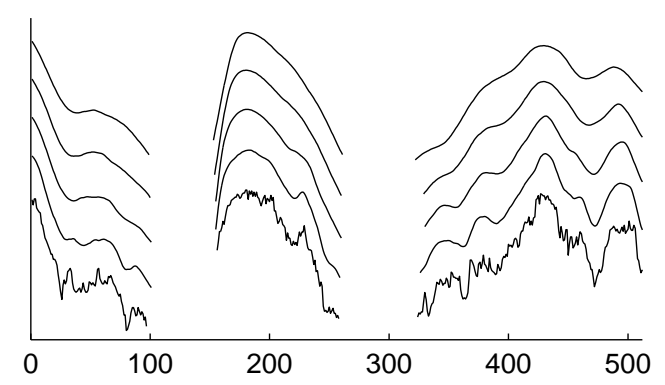

Fig. 4. Influence of the crystallization parameter $b$ on the shape of a thin film profile. Image profiles with parameters $b=0,5,10,15,20$ are drawn.

Several histograms are shown here capable of crystallization detection. The parameter $b$ can be deduced from the pairs of the parameters $\alpha$ and $\beta$. All of them are drawn in the following figures and discussed. In every graph, values of the features for the first three levels of decomposition are shown. There is a simple mnemonic rule for a quick orientation in the graphs. Every mark used for its level has equal number of edges, i.e. level one is denoted by $\circ$, level two by $\times$ and level three by $\triangle$. In the left, resp. right, graph, dependence of the parameter $\alpha$, resp. $\beta$, on the crystallization parameter $b$ is shown. All the parameters $\alpha$ are normalized so, that they can be compared with the first level.

$\mathrm{CM}$ for the following three histograms is constructed so, that a CM is calculated for coefficients from the horizontal sub-image and their neighbors in distance [0 1]. The vertical sub-image is processed analogously and the CMs are summed up.

Histogram $M 1$, modeled as symmetrical, is used in Fig. 5. The histogram tails are formed by the neighbors, which tend to have the same sign, thus forming a continuing slope. The right, resp. left side of the histogram corresponds to positive, resp. negative slopes. It can be seen that when the scale of the blurring kernel passes through a characteristic scale of a particular level, the parameter $\alpha$ reaches 
an almost steady value. Inspecting the parameter $\beta$ after passing the characteristic scale, it is found to be growing. At that stage, the thin film surface appears smoother compared to the scale of the analyzing wavelet. There are more less steep slopes.
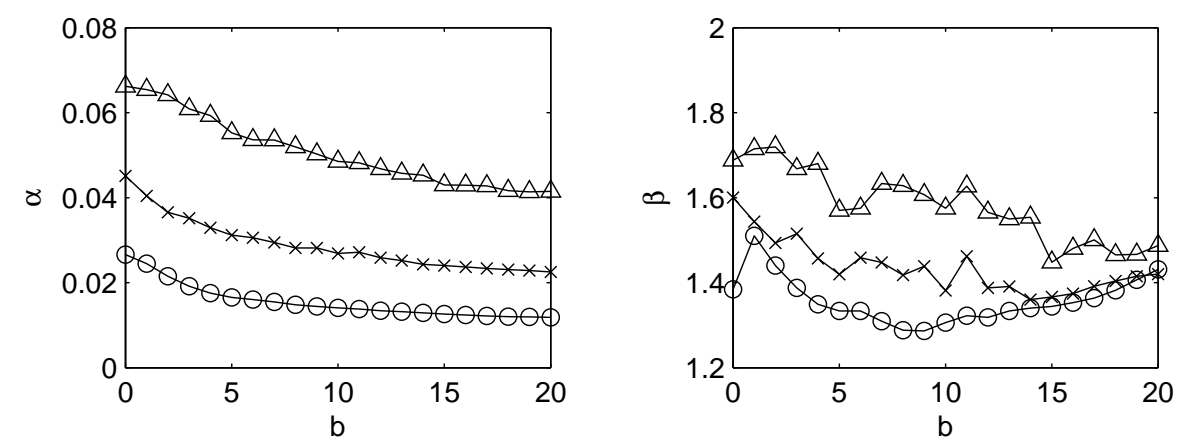

Fig. 5. Parameters $\alpha$ and $\beta$, dependent on the crystallization parameter $b$. Histogram $M 1$ of both horizontal and vertical coefficients with shifts in perpendicular direction and symmetrical modeling are used. o - first level, $\times$ - second level, $\triangle$ - third level.

Before reaching the characteristic scale, $\beta$ decreases. Lower $\beta$ usually means a sharper structure. However, this phenomenon is accompanied with fall of $\alpha$, which suggests presence of coefficients with lower magnitudes. Thus, a caution is necessary when interpreting the values. Other slight fluctuations of $\beta$ can be regarded as a measurement deviation. The characteristic scale, where the valley in the parameter $\beta$ appears, is probably significant due to transition of a rugged structure to a monotone slope at this scale. At this point, there are more plateaus, contributing to zero coefficients of the marginal histogram and pushing the parameter $\beta$ to lower values.

Features based on histogram $S 1$, modeled as symmetrical, are depicted in Fig. 6 . The histogram is analogous to $M 1$, but the slopes at both neighbors have strictly the same sign. For small $b$, the parameter $\beta$ is relatively large, since the tops and bottoms of the structure are narrow and thus not contributing to the coefficients close to zero. With larger $b$, peaks become smoother, with less steep tops and longer steeper walls. They contribute more to the center of the histogram and to its tails, thus decreasing $\beta$. The plateaus from the previous Figure are not so significant here, because the slopes must have the same sign.

In Fig. 7, histogram $S 3$ is used. Parameters of the left halves of the histogram are drawn. The histogram is analogous to $M 2$, but the slopes at both neighbors have strictly different signs. It is interesting to notice that the contrast $\alpha$ of a strictly concave behavior has the tendency also to rise for the initial smoothing, see level 3. Modest smoothing probably enhances the striations, which are detected by this feature. Parameter $\beta$ generally rises, as expected, although with a substantial fluctuation. The fluctuation is caused by a small number of coefficients available for 

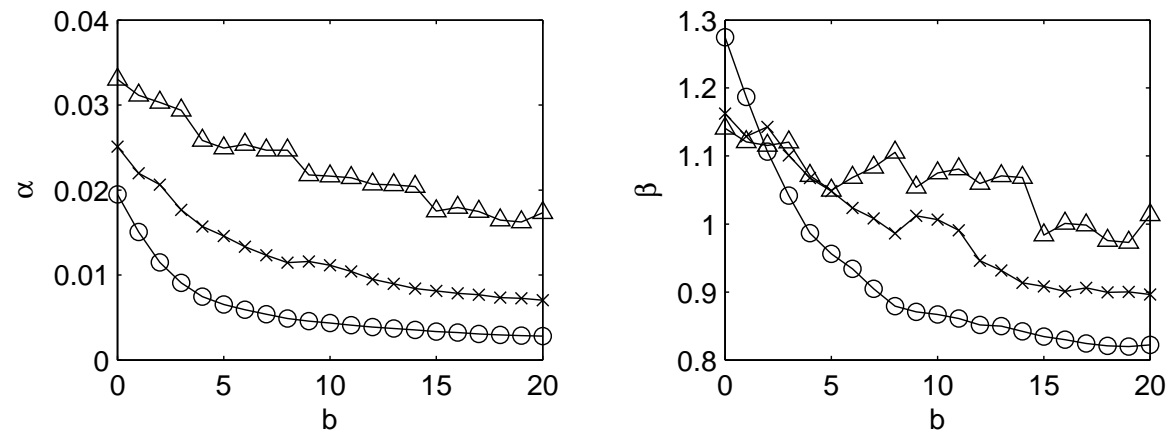

Fig. 6. Parameters $\alpha$ and $\beta$, dependent on the crystallization parameter $b$. Histogram $S 1$ of both horizontal and vertical coefficients with shifts in perpendicular direction and symmetrical modeling are used. $\circ$ - first level, $\times$ - second level, $\triangle$ - third level.

the histogram fitting. Generally, the main reason, which causes high fluctuations of the parameter $\beta$, is worse fit of the model (1) to the particular histogram. As a consequence, it causes a numerical instability of the fitting procedure. Certain role is also played by discretization errors.
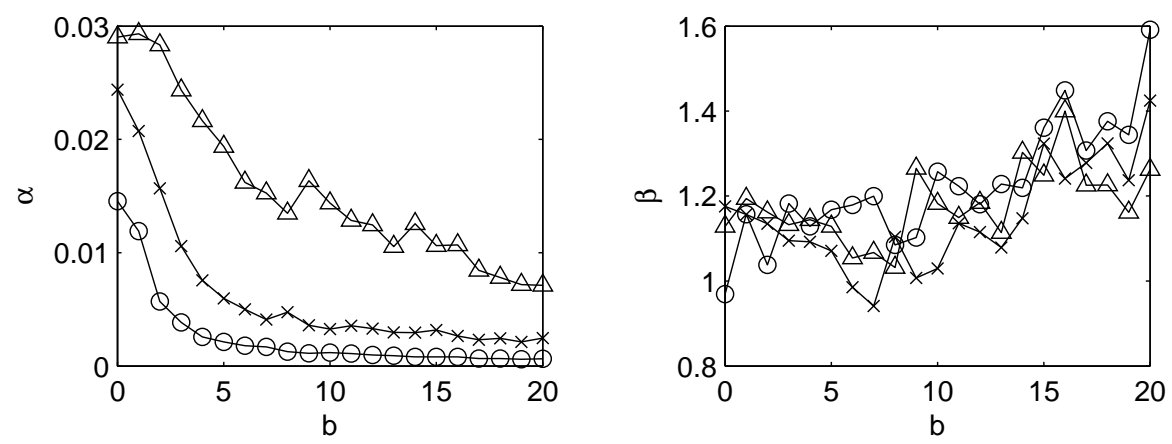

Fig. 7. Parameters $\alpha$ and $\beta$, dependent on the crystallization parameter $b$. Histogram $S 3$ of both horizontal and vertical coefficients with shifts in perpendicular direction and modeling of the left half are used. $\circ$ - first level, $\times$ - second level, $\triangle$ - third level.

The following two histograms are constructed analogously to the previous histograms, but the distance between neighbors used in the CMs is [1 0]. The histogram $S 1$ for this CM, modeled as symmetrical, also proved powerful for crystallization detection, see Fig. 8. Only slopes with the same sign, thus forming a continuing edge, contribute to this histogram. Similar comments as for Fig. 6 apply also here.

Features based on the sum of histograms $S 0$ and $S 2$, modeled as symmetrical, 

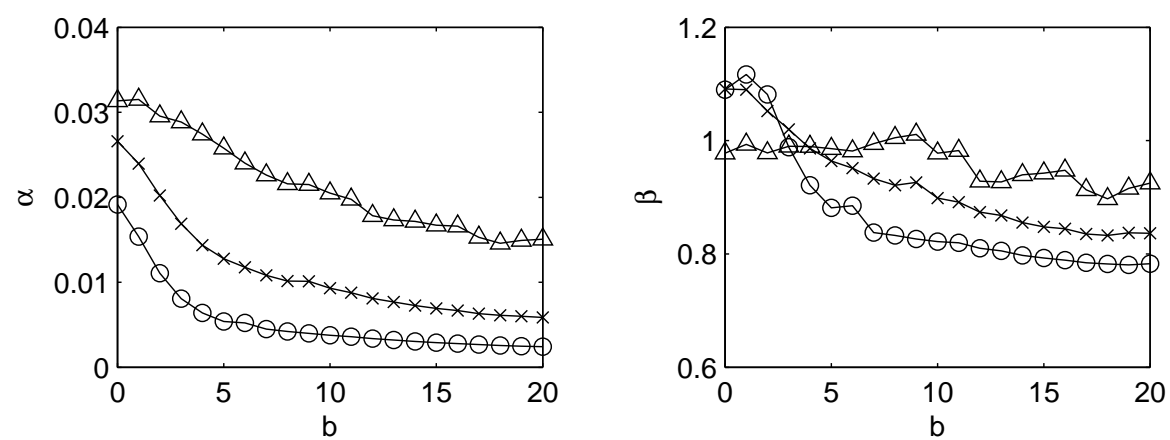

Fig. 8. Parameters $\alpha$ and $\beta$, dependent on the crystallization parameter $b$. Histogram $S 1$ of both horizontal and vertical coefficients with shifts in parallel direction and symmetrical modeling are used. $\circ$ - first level, $\times$ - second level, $\triangle$ - third level.

are shown in Fig. 9. The histogram tails are formed by the neighbors, where one of them has a significant gradient and the other one very small or zero gradient. It corresponds to an edge ending (edge is meant here in the sense of a non-zero slope). The parameters $\alpha$ and $\beta$ indicate that development of curvature of the thin film slopes is qualitatively significant only up to a certain parameter $b$. Afterwards, only contrast continues decreasing.
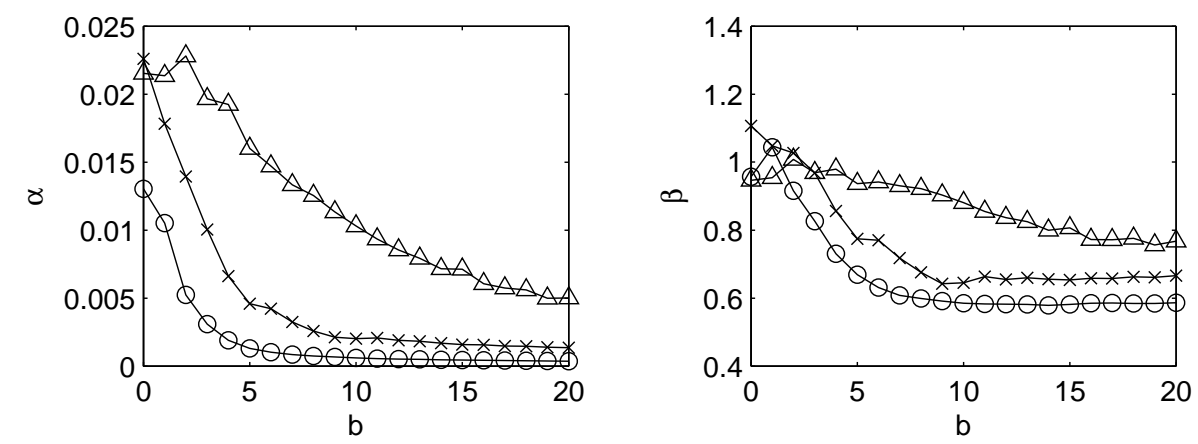

Fig. 9. Parameters $\alpha$ and $\beta$, dependent on the crystallization parameter $b$. Sum of histograms $S 0, S 2$ of both horizontal and vertical coefficients with shifts in parallel direction and symmetrical modeling are used. $\circ$ - first level, $\times$ - second level, $\triangle$ - third level.

The last histogram is again a sum of CMs for the horizontal and the vertical sub-images. Coefficients from the sub-images at the next coarser level and at the same spatial position are used as pairs to the CMs. In Fig. 10, features are shown based on histogram $S 1$, modeled as symmetrical. The histogram tails are formed 
by the neighbors in scale, where the horizontal slopes at one scale have the same magnitudes also in finer resolution. Again, similar comments as for Fig. 6 apply here, although the features capture different characteristics.
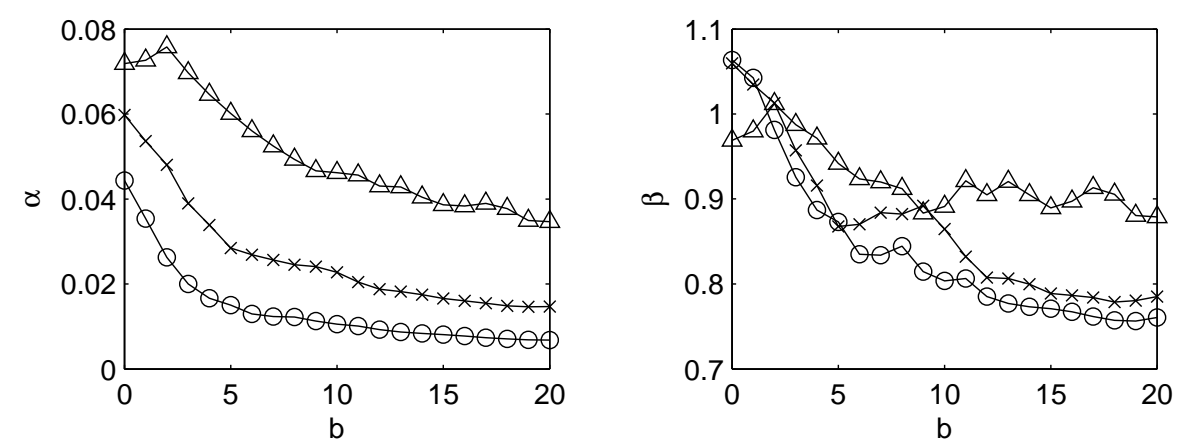

Fig. 10. Parameters $\alpha$ and $\beta$, dependent on the crystallization parameter $b$. Histogram $S 1$ of both horizontal and vertical coefficients from neighboring scales and symmetrical modeling are used. $\circ$ - first level, $\times$ - second level, $\triangle$ - third level.

\section{Conclusions}

Ability of wavelets to describe structure of thin films is investigated in this paper. A unique set of features based on wavelet coefficients and their 1D and 2D histograms has been derived and used for thin film description. All the used descriptors have been interpreted and their tight correspondence with the thin film morphology has been shown. A computer experiment has been performed to demonstrate that the features are robust and easy to interpret.

A few improvements are planned for the future, including speeding up the calculations and decreasing dependence of some of the features, taking into account a particular application.

\section{Acknowledgments}

The work is a part of research intent No. MSM 113200002. The support of the grant GAUK-173/2003 of the Grant Agency of Charles University in Prague is gratefully acknowledged.

J. Boldyš is currently working in the Laboratory of Media Technology, Department of Automation and Systems Technology, Helsinki University of Technology, Otaniementie 17, 02150 Espoo, Finland. 


\section{References}

[1] J. Serra: Image Analysis and Mathematical Morphology. Academic Press, London, 1982.

[2] B.D. Ripley: Spatial Statistics. John Wiley and Sons Inc., New York, 1981.

[3] H. Ebeling and G. Wiedenmann: Phys. Rev. E. 47 (1993) 704.

[4] C.L.Y. Yeong and S. Torquato: Phys. Rev. E. 57 (1998) 495.

[5] R. Hrach, D. Novotný, S. Novák, and J. Pavlík: Thin Solid Films. 433 (2003) 135.

[6] M. Sonka, V. Hlavac, and R. Boyle: Image Processing, Understanding, and Machine Vision. PWS, Boston, 1998.

[7] G. Strang and T. Nguyen: Wavelets and Filter Banks. Wellesley-Cambridge Press, 1996.

[8] M. Misiti, Y. Misiti, G. Oppenheim, and J.-M. Poggi: Wavelet Toolbox User's Guide. The MathWorks, Inc., 2002.

[9] J. Portilla and E.P. Simoncelli: Int. J. Computer Vision. 40 (2000) 49.

[10] S.G. Mallat: IEEE Trans. Pattern Analysis and Machine Intelligence. 11 (1989) 674.

[11] G. Van de Wouver, P. Scheunders, and D. Van Dyck: IEEE Trans. Image Processing. 8 (1999) 592.

[12] U. Grenander and A. Srivastava: IEEE Trans. Pattern Analysis and Machine Intelligence. 23 (2001) 424.

[13] J. Boldyš: Multiscale Semicontinuous Thin Film Image Analysis. PhD thesis, Charles University in Prague, Faculty of Mathematics and Physics, 2004. 